\title{
Is a Layered Medium One Dimensional?
}

\author{
Ping Sheng ${ }^{1,2}$ and Zhao-Qing Zhang ${ }^{2}$ \\ ${ }^{1}$ Exxon Research and Engineering Company, Route 22 East, Annandale, New Jersey 08801 \\ ${ }^{2}$ Department of Physics, Hong Kong University of Science and Technology, Clear Water Bay, Kowloon, Hong Kong
}

(Received 28 February 1994)

\begin{abstract}
In the study of wave transport, a randomly layered medium is generally considered one dimensional due to its localizing character in the layering direction. However, we show that when the system is perturbed by the addition of a small amount of isotropic randomness, wave propagation in the direction parallel to the layers can exhibit significant leakage out of the localizing layers. Numerical study of this phenomenon in 2D anisotropic models reveals the existence of wave-energy tunneling via evanescent waves in the layering direction, which gives rise to finite-size scaling behavior intermediate between 1D and $2 \mathrm{D}$.
\end{abstract}

PACS numbers: $42.25 . \mathrm{Bs}, 03.40 . \mathrm{Kf}, 63.50 .+\mathrm{x}$

A randomly layered medium has identical wave localization behavior as a $1 \mathrm{D}$ chain for waves propagating in the layering direction, i.e., the direction perpendicular to the layers. Moreover, localized eigenstates in the layering direction define "channels," in which the wave energy is confined for waves propagating parallel to the layers. Thus, the confinement of wave energy in the layering direction, regardless of the propagation direction, is indicative of the 1D character of a randomly layered medium.

In this Letter, we show that the confinement of wave energy in channels is unstable against small perturbation of additive isotropic randomness. Physically, a layered medium with additive isotropic randomness describes, for example, the Earth's subsurface of random superlattices with inhomogeneities. In the presence of isotropic randomness, wave energy initially localized (in the layering direction) in a particular channel can be significantly delocalized via the mechanism of tunneling between the channels. However, the tunneling is not symmetric: There exist dominant channels that attract wave energy from the others [1]. The resulting wave amplitude distribution is thus nonergodic and exhibits a finite-size scaling behavior that is intermediate between 1D and 2D.

Consider the 2D tight-binding Hamiltonian

$$
H=\sum_{\alpha} \varepsilon_{\alpha} a_{\alpha}^{\dagger} a_{\alpha}+t \sum_{[\alpha, \beta]} a_{\alpha}^{\dagger} a_{\beta}
$$

where $t=1$ is the hopping matrix element, $\alpha, \beta$ are the site indices of a simple square lattice on the $x-z$ plane, $[\alpha, \beta]$ indicates $\alpha$ and $\beta$ are nearest neighbors, and

$$
\varepsilon_{\alpha}=\eta_{z}+\gamma_{\alpha}
$$

is composed of two components: $\eta_{z}$ is a number that is a constant for all $x$ and fixed $z$, but that varies randomly as $z$ varies with a flat distribution

$$
P_{1}\left(\eta_{z}\right)= \begin{cases}1 / W_{1} & \left|\eta_{z}\right| \leq W_{1} / 2 \\ 0 & \text { otherwise. }\end{cases}
$$

$\gamma_{\alpha}$ is a random number that varies independently from site to site with a flat distribution

$$
P_{2}\left(\gamma_{\alpha}\right)= \begin{cases}1 / W_{2} & \left|\gamma_{\alpha}\right| \leq W_{2} / 2 \\ 0 & \text { otherwise }\end{cases}
$$

In other words, $\eta_{z}$ represents the random layerings and $\gamma_{\alpha}$ the additive, isotropic randomness. In the limit of $W_{2}=0$, we have a randomly layered system. Along the layering direction ( $z$ axis), eigenvalues and eigenfunctions of $H$ can be simply obtained by the diagonalization of $H$. We would like to study the case where the layering randomness is strong, i.e., $W_{1}$ large, in which the eigenfunctions will be highly localized with a localization length $\xi_{\perp} \cong 1-2$ layers. Even in this case, however, an important point to be noted is that the eigenfunctions can still have significant spatial overlaps; although in the absence of isotropic randomness they are orthogonal to each other in the sense that if initially only a particular eigenfunction is excited, waves will propagate in the $x$ direction as in a waveguide, i.e., confined in the $z$ direction. We define the localized eigenfunctions as channels for waves propagating in the $x$ direction.

For a layered 2D system, the wave energy can be written as

$$
E=E_{z}+2 \cos k_{x} a_{0},
$$

where $a_{0}=1$ is the lattice constant. By definition, each channel is associated with a unique $E_{z}$. When $\mid E-$ $E_{z} \mid>2, k_{x}$ is imaginary and the channel is denoted "evanescent." When $\left|E-E_{z}\right|<2$ and $k_{x}$ is real, the channel is denoted "propagating." In a geometry where the sample [defined by Eqs. (1)-(4)] is connected to the left and to the right with perfect leads, i.e., $W_{1}=W_{2}=$ 0 , the evanescent and propagating modes describe the incident and transmitted waves.

In the presence of a small amount of isotropic randomness, e.g., $W_{2}=0.1 W_{1}$, the localizing character of the randomly layered media $[2,3]$ is not appreciably altered for waves propagating in the $z$ direction: When $W_{1}=15$ and $W_{2}=1.5, \quad \xi_{\perp}$ is increased to $\sim 1.4$ from its value of $\sim 1.2$ at $W_{2}=0$. This calculation can be carried out numerically by using the recursive Green's function approach [4] on a strip of width $M$ in the $x$ direction and length $L \gg M$ in the $z$ direction. The value of $\xi_{\perp}$ is found to saturate at $\sim 1.4$ when $M$ is increased to $\sim 50$. For waves 
propagating in the $x$ direction, however, qualitatively new phenomenon is found. The same recursive Green's function approach can be applied here, with width $M$ of the strip now defined to be in the $z$ direction, and the length $L$ in the $x$ direction. For $L$ sufficiently large, $\xi_{\|}$can be accurately obtained for each single configuration. Since one can obtain a localization length for each channel, the $\xi_{\|}$for each configuration is given by the largest localization length among all the channels in that configuration [5]. As the layering randomness $\eta_{z}$ is changed from one configuration to the next, the values of $\xi_{\|}$thus obtained form a peaked distribution. We choose the most probable value of the distribution as the representative value of $\xi_{\|}$.

For a $1 \mathrm{D}$ strip of width $M$ in the $z$ direction, it is generally the case that

$$
\xi_{\|} \propto M / W_{2}^{2},
$$

for $\xi_{\|} \gg M$. If the wave energy is predominantly confined in individual channels, it is plausibly expected that if a particular channel has a $z$ direction localization length $\xi_{\perp}$, then its $\xi_{\|}$should be

$$
\xi_{\|} \propto \xi_{\perp} / W_{2}^{2},
$$

in the limit of $\xi_{\|} \gg \xi_{\perp}$. This turns out not to be the case for the simple reason that the introduction of isotropic randomness can introduce an interchannel exchange of the wave energy. This is clearly demonstrated in Fig. 1 for two channels, where the incident wave into channel " $B$ " is shown to be progressively transferred to channel " $A$ " (which is in fact spatially far away from channel $B$ ) until, in the tail region, channel $B$ decays at the same rate as channel $A$. For channel $B$ there is thus a twosegment behavior in its spatial decay: The initial decay involves not only the localizing effect of the isotropic randomness, but also the transfer of energy to channel $A$; whereas in the second segment, the behavior of channel $B$ is controlled by channel $A$. If the wave energy were initially injected into channel $A$, then only the second segment of $B$ would be evident. In this example, $A$ is clearly seen to be the dominant channel. In fact, for each configuration there is always a dominant channel, which

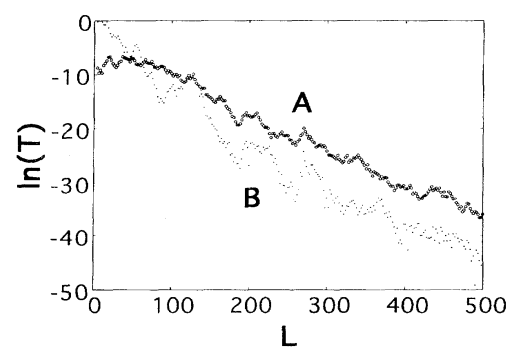

FIG. 1. Spatial decay of two channels along the $x$ direction, with wave incident on channel $B$. Note the two-segment behavior of the $B$-channel decay. $T$ denotes the amplitude transmission coefficient. Here $M=10, W_{1}=15, W_{2}=1.5$, and the result is from a single configuration calculation. is also the one whose localization length is the largest and which therefore defines the localization length for that particular configuration. To study the behavior of wave propagation parallel to the layers is thus equivalent to the study of the dominant channel behavior.

What determines the dominant channel behavior? Here a search for dependence on $\xi_{\perp}$ [as given by Eq. (6)] yields no perceivable correlation. More relevant is the parameter $\boldsymbol{v}_{x}=\left|\partial E_{x} / \partial k_{x}\right|$. In Fig. 2 the $v_{x}$ distribution of the dominant channel is plotted. A clear trend for the dominant channel to have a large $v_{x}$ is seen. This correlation is intuitively plausible because a state with a larger $v_{x}$ should take longer to slow down and be localized than the one with a smaller $v_{x}$. However, even for $v_{x} \cong 2$, the maximum value, the amount of wave intensity in the dominant channel can still be relatively small at a given value of $x$. To study this aspect of the problem quantitatively, we define the occupation ratio of a particular channel as

$$
\theta_{i}=\frac{1}{M_{c}}\left\langle\sum_{j} \frac{\left|t_{j i}\right|^{2}}{\sum_{i}\left|t_{j i}\right|^{2}}\right\rangle_{x},
$$

where $M_{c}$ is the number of propagating channels in the left or right lead attached to the sample, $j$ is the index for the incident channels, $i$ denotes the output (measurement) channel, $t_{j i}$ is the transmission amplitude between channel $j$ and channel $i$ as calculated by using the recursive Green's function approach, and $\langle\cdots\rangle_{x}$ means averaging along the $x$ positions in the asymptotic regime where the statistics of $\theta_{i}$ is stationary. Apart from minor fluctuations, the final answer for the occupation ratio has been tested to be independent from the initial injection channel. In Fig. 3 we plot the distribution of the occupation ratio of the dominant channel for sample configurations with $M=20$ and 50. For $M=20$, it is seen that while there are some cases where the dominant channel's occupation ratio is in the range of $0.8-0.9$, the most probable occupation ratio of the dominant channel is on the order of $0.2-0.3$, i.e., $(70-80) \%$ the wave energy at a given $x$ is spread to the other channels. As $M$

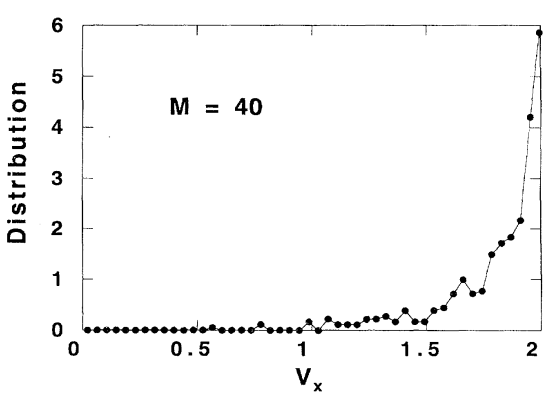

FIG. 2. The $v_{x}$ distribution of the dominant channel in each configuration. Here $M=40, W_{1}=15, W_{2}=1.5$, and the result comprises 460 configurations. 


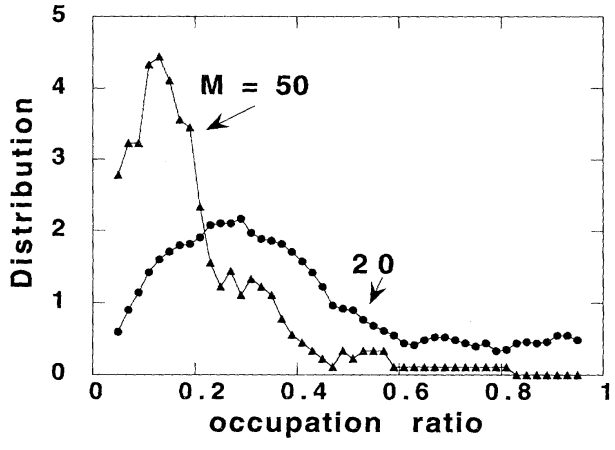

FIG. 3. Distributions of the dominant-channel occupation ratio for $M=20$ and 50. Here $W_{1}=15, W_{2}=1.5$, and the distribution for the $M=20$ case has 460 configurations, and the distribution for the $M=50$ case has 90 configurations.

is increased to 50 , the most probable occupation ratio decreases to $\sim 0.1$, with a corresponding decrease in the probability for occupation ratio higher than 0.5 .

The key to the understanding of this delocalizing behavior lies in the evanescent channels. In the example shown in Fig. 1, while $A$ and $B$ are the only two propagating channels, there are many other evanescent channels which overlap spatially with $A$ and $B$ and with each other. These channels cannot carry energy in the $x$ direction, but they provide the means of interchannel energy transfer in the presence of isotropic randomness. Wave energy can thus "tunnel" from one channel to the next, resulting in significant delocalization of the wave energy in the $z$ direction. As $M$ increases, the total number of channels increases proportionally, and interchannel tunneling is expected to become progressively more efficient as the average energy spacing between the $E_{z}$ 's decreases, so that the overlap between the $E_{z}$ levels increases.

Because of the existence of the dominant channel, the distribution of wave energy between the propagating (nonevanescent) channels is never ergodic. A clean demonstration of this fact is via the calculation of the average number of occupied channels

$$
N_{\mathrm{oc}}\left(M_{c}\right)=\frac{1}{M_{c}} \sum_{i} \frac{\left[\sum_{j}\left|t_{i j}\right|^{2}\right]^{2}}{\sum_{j}\left|t_{i j}\right|^{4}},
$$

which is recognized to be the inverse of the average participation ratio. A plot of $N_{\mathrm{oc}}\left(M_{c}\right)$ for these different models is shown in Fig. 4. Data for the case under consideration are denoted by open circles, whereas crosshatched squares and open triangles denote the cases of the $2 \mathrm{D}$ isotropic model and the $2 \mathrm{D}$ anisotropic hopping model [6], respectively. In the anisotropic hopping model, $\varepsilon_{\alpha}$ n Eq. (1) contains only the $\gamma_{\alpha}$ term, but the hopping matrix element $t$ is anisotropic. It is seen that models $B$ and $C$ both exhibit linear dependence on the number of propagating channels $M_{c}$, as dictated by ergodicity,

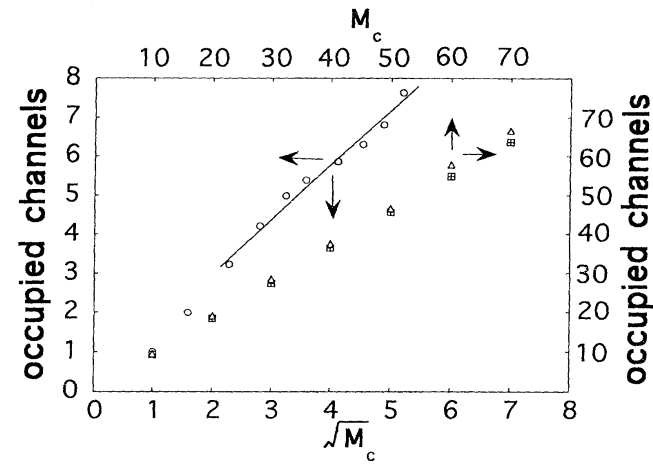

FIG. 4. Number of occupied channels plotted as a function of $M_{c}$, the number of propagating channels. Whereas the isotropic model $(\boxplus)$ and the anisotropic hopping model $(\Delta)$ exhibit linear dependence on $M_{c}$ (both have $W_{1}=0, W_{2}=1.5$, with 1 configuration; the anisotropic model has $t_{x}=1, t_{z}=0.1$ ), the presence of isotropic randomness in a randomly layered medium (O) results in a $\sqrt{M_{c}}$ variation. It should be noted that $M_{c}=20$ in the present model means $M \approx 80-100$, whereas for the isotropic model $M_{c}$ corresponds roughly with $M$. Therefore the sizes of the actual calculations in all three models are comparable. Here $W_{1}=15, W_{2}=1.5$, and 460 configurations are used in averaging for $M=10,15,20,25$, and 30 . 80 configurations are used in averaging for $M=70,75$, and 80 , and 30 configurations are used for $M=85$. The rms deviation of the overall data is about $10 \%$.

whereas model $A$ shows an excellent $\sqrt{M_{c}}$ dependence. The same $\sqrt{M_{c}}$ dependence is also observed for the most probable value of the $\xi_{\|}$distribution. If a "truly $1 \mathrm{D}$ " behavior is defined as where the wave energy is always confined in each channel so that the resulting dependence on $M_{c}$ is flat, then Fig. 4 shows our case to be intermediate between $1 \mathrm{D}$ and $2 \mathrm{D}$. Of course, the $\sqrt{M_{c}}$ dependence is not expected to be maintained beyond the limit of $M_{c}=$ the localization length of the isotropic model with randomness $W_{2}$. However, for small $W_{2}$ this upper bound can be transcendentally large. The precise origin of the $\sqrt{M_{c}}$ behavior is under further study. Results will "be reported in another publication.

The implication of our findings for the time evolution of a localized pulse is as follows. For a given channel, wave tunneling is analogous to a scattering extinction effect. The energy of a localized pulse is therefore expected to leak out of the channel region defined by $\xi_{\perp}$, with a time constant determined by the ratio $W_{2} / W_{1}$. In the limit of small $W_{2} / W_{1}$, the decay is expected to be transcendentally slow due to the fact that the energy spreading occurs mostly in the exponential tail region of the intensity distribution. However, while the intensity in the tail region is arguably small, the large value of the cutoff for the $\sqrt{M_{c}}$ behavior (the isotropic $2 \mathrm{D} \xi$ ) can mean that a significant fraction of the total intensity will eventually be outside of the initial channel. As an example, for $M_{c}=50$ an estimated $(20-50) \%$ of the total intensity lies outside of the initial channel. 
One of the authors (P.S.) wishes to thank the Institute for Advanced Study for a visiting appointment in the 1992-93 academic year, during which this work was started and stimulated by numerous discussions with Thomas Spencer. He also wishes to thank George Papanicolaou for many useful discussions.

[1] The fact that the tunneling need not be symmetric can easily be seen from the fact that $t_{i j} \neq t_{j i}$, in general. This can be demonstrated in the case of two intermediate scatterings by impurity potentials denoted as $\nu_{n}$ and $\nu_{m}$ :

$$
\begin{aligned}
\int \phi_{j}\left(z^{\prime}\right) \nu_{n}\left(z^{\prime}\right) G_{n m}\left(z^{\prime}, z\right) \nu_{m}(z) \phi_{i}(z) d z^{\prime} d z & \\
& \neq \int \phi_{i}\left(z^{\prime}\right) \nu_{n}\left(z^{\prime}\right) G_{n m}\left(z^{\prime}, z\right) \nu_{m}(z) \phi_{j}(z) d z^{\prime} d z,
\end{aligned}
$$

where $\phi_{i}$ is the wave function of the $i$ th channel and $G$ denotes the Green's function. Only in the case of single scattering does $t_{i j} \neq t_{j i}$.

[2] W. Xue, P. Sheng, Q. J. Chup, and Z. Q. Zhang, Phys. Rev. Lett. 63, 2837 (1989).

[3] V. Freilikher and Yu. Tarasov, IEEE Trans. AP 39, 197 (1991).

[4] P. A. Lee and D. Fisher, Phys. Rev. Lett. 47, 882 (1981).

[5] Localization length is usually defined for a momentum eigenchannel. Since a real space channel may be expressed as the linear combination of momentum eigenchannels, the localization length of a real space channel is therefore dominated (at large distances) by the largest localization length among the eigenchannels. That is defined to be the localization length of the real space channel.

[6] W. Apel and T. M. Rice, J. Phys. C 16, L1151 (1983); Q. Li, C. M. Soukoulis, E. N. Economou, and G. S. Grest, Phys. Rev. B 40, 2825 (1989). 